\title{
Effect of Proton Irradiation Followed by Hindlimb Unloading on Bone in Mature Mice: A Model of Long-Duration Spaceflight
}

\author{
Shane A. Lloyda, ${ }^{a}$, Eric R. Bandstra ${ }^{b,}{ }^{*}$, Jeffrey S. Willey ${ }^{c}$, Stephanie E. Riffle ${ }^{b}$, Leidamarie \\ Tirado-Lee $^{b}$, Gregory A. Nelson ${ }^{d}$, Michael J. Pecaut ${ }^{d}$, and Ted A. Bateman ${ }^{e}$ \\ aDivision of Musculoskeletal Sciences, Department of Orthopaedics and Rehabilitation, Penn \\ State College of Medicine, 500 University Drive, Hershey, PA, USA, 17033 \\ ${ }^{b}$ Department of Bioengineering, Clemson University, 501 Rhodes Research Center, Clemson, \\ SC, USA, 29634 \\ 'Section of Molecular Medicine and Department of Radiation Oncology, Comprehensive Cancer \\ Center, Wake Forest School of Medicine, Medical Center Boulevard, Winston-Salem, NC, USA, \\ 27157 \\ dDepartment of Radiation Medicine, Loma Linda University and Medical Center, 11234 Anderson \\ Street Loma Linda, CA, USA, 92354 \\ eDepartments of Biomedical Engineering and Radiation Oncology, University of North Carolina at \\ Chapel Hill, 152 MacNider Hall, Chapel Hill, NC, USA, 27599
}

\section{Abstract}

Bone loss associated with microgravity unloading is well documented; however, the effects of spaceflight-relevant types and doses of radiation on the skeletal system are not well defined. In addition, the combined effect of unloading and radiation has not received much attention. In the present study, we investigated the effect of proton irradiation followed by mechanical unloading via hindlimb suspension (HLS) in mice. Sixteen-week-old female C57BL/6 mice were either exposed to $1 \mathrm{~Gy}$ of protons or a sham irradiation procedure ( $\mathrm{n}=30 /$ group). One day later, half of the mice in each group were subjected to four weeks of HLS or normal loading conditions.

Radiation treatment alone (IRR) resulted in approximately $20 \%$ loss of trabecular bone volume fraction (BV/TV) in the tibia and femur, with no effect in the cortical bone compartment. Conversely, unloading induced substantially greater loss of both trabecular bone (60-70\% loss of $\mathrm{BV} / \mathrm{TV}$ ) and cortical bone (approximately $20 \%$ loss of cortical bone volume) in both the tibia and femur, with corresponding decreases in cortical bone strength. Histological analyses and serum chemistry data demonstrated increased levels of osteoclast-mediated bone resorption in unloaded mice, but not IRR. HLS+IRR mice generally experienced greater loss of trabecular bone volume fraction, connectivity density, and trabecular number than either unloading or irradiation alone. Although the duration of unloading may have masked certain effects, the skeletal response to irradiation and unloading appears to be additive for certain parameters. Appropriate modeling of the environmental challenges of long duration spaceflight will allow for a better understanding of

(C) 2012 Elsevier Inc. All rights reserved.

Corresponding Author: Ted A. Bateman, Department of Biomedical Engineering, University of North Carolina, 140 MacNider Hall, Chapel Hill, NC, USA 27599, Telephone: 919-966-1175, bateman@unc.edu.

*These authors contributed equally to this work.

Publisher's Disclaimer: This is a PDF file of an unedited manuscript that has been accepted for publication. As a service to our customers we are providing this early version of the manuscript. The manuscript will undergo copyediting, typesetting, and review of the resulting proof before it is published in its final citable form. Please note that during the production process errors may be discovered which could affect the content, and all legal disclaimers that apply to the journal pertain. 
the underlying mechanisms mediating spaceflight-associated bone loss and for the development of effective countermeasures.

\section{Keywords}

bone; radiation; unloading; hindlimb suspension; spaceflight

\section{INTRODUCTION}

Astronauts on missions beyond Earth's orbit will be required to live and work in a microgravity environment for long periods of time. The response of the skeletal system to mechanical unloading has been studied in astronauts after four to six month missions on the International Space Station (ISS) [1]. Skeletal deterioration has been documented in these individuals in areas that are prone to fracture, including the vertebrae and hip. Bone loss observed in this population translated into reductions in estimated bone strength indices [1], suggesting that astronauts could be at a higher risk for fracture during or after extended missions. For ground-based modeling, hindlimb suspension (HLS) is a widely accepted rodent model that is able to simulate the mechanical unloading and cephalic-fluid shifts encountered in microgravity [2]. After two to three weeks of HLS, skeletally mature mice experience reductions in bone volume fraction in the distal femur on the order of what a human would expect to lose following four to six months of spaceflight $[3,4]$.

During extended missions, astronauts will also be exposed to both solar and cosmic radiation. Due to the shielding effect of the Earth's magnetosphere, the type and dose of radiation encountered will be significantly different than those experienced on the ISS, which is located in low Earth orbit [5]. Multiple spaceflight-relevant types of radiation have been documented as having negative effects on trabecular bone [6]. For Mars missions, cosmic rays (containing heavy ions and protons) and solar particle events (SPEs; composed mainly of protons) are the primary concern for radiation exposure [7-9]. SPEs occur randomly and can deliver a relatively high dose (up to $2 \mathrm{~Gy}$ ) over a short period of time [8, $10,11]$. As a basis for comparison, $2 \mathrm{~Gy}$ is equal to a single radiation fraction commonly administered to a patient undergoing radiotherapy for a solid tumor [12]. Although spacecraft shielding can effectively reduce radiation exposure, the warning provided by current surveillance mechanisms may not allow for complete protection during extravehicular activities [13]. Even if astronauts were sheltered behind a mass of $5 \mathrm{~g} / \mathrm{cm}^{2}$ (e.g., $1.9 \mathrm{~cm}$ of aluminum or $5 \mathrm{~cm}$ of water), severe SPEs, such as those observed in August 1972 and October 1989, could deliver whole-body radiation doses approaching 2 Gy [14]. Given the duration of future missions planned by NASA to nearby asteroids and Mars [15], a dose of approximately 1 Gy proton radiation is a realistic possibility and should be used to develop mission-safety protocols [16-18]. Previous studies in our laboratory have demonstrated significant, long-term loss of trabecular microstructure at multiple sites following doses as low as 1 Gy protons [19] or 2 Gy X-rays [20].

While bone loss associated with unloading has been well characterized, the effects of radiation on the skeletal system are not as well defined. Furthermore, there are few published reports detailing the effects of both radiation exposure and unloading on bone, although emerging evidence suggests that these two factors may interact to result in increased bone loss. In a study by Yumoto and colleagues, irradiation of skeletally mature mice following a short period of HLS was shown to result in greater suppression of osteoblast-mediated bone formation than either treatment alone [21]. Finite element computer modeling predicted increased loss of skeletal integrity and strength in the lumbar vertebrae of irradiated animals subjected to musculoskeletal disuse [22]. An understanding 
of how the combination of these factors affects the skeletal system is necessary to facilitate appropriate planning for long-duration missions. In addition, the development of effective countermeasures will require an understanding of what factors make meaningful contributions to skeletal deterioration in space. In pursuit of this end, the present study aims to utilize proton irradiation followed by unloading via HLS to investigate the combined effects of these modeled aspects of the spaceflight environment. Protons are a low linear energy transfer (LET) type of radiation, meaning that they tend to penetrate deeper in to tissue and deposit less energy compared to high LET types of radiation, such as larger carbon or iron particles $[12,23]$. Previous studies have not investigated the effect of low LET radiation and unloading [22]. It is hypothesized that the combination of radiation and unloading will lead to greater bone loss than will either challenge separately.

\section{MATERIALS AND METHODS}

\subsection{Animals}

Female C57BL/6 mice (Taconic Farms; Germantown, NY) were shipped to the Loma Linda University Medical Center (Loma Linda, CA) at fifteen weeks of age and acclimatized for 1 week under standard vivarium conditions. Animals were grouped by mass (5 groups, $\mathrm{n}=15-$ 17/group). A detailed description of the treatment groups is presented in Table 1. All mice were provided with standard laboratory rodent chow and water ad libitum. All animal procedures were approved by the Institutional Animal Care and Use Committee at both Loma Linda University and Clemson University (Clemson, SC).

\subsection{Irradiation}

Two groups of animals were irradiated (IRR), while the remaining experimental animals were subjected to a sham irradiation procedure (i.e., non-irradiated controls; NR). A separate group of baseline mice were killed at the start of the study and were not subjected to any of these procedures. Immediately prior to irradiation, each mouse (test and sham-irradiated) was placed individually into a rectangular plastic box $(30 \times 30 \times 85 \mathrm{~mm})$ with air holes, as described previously [24]. A maximum of 6 mice were irradiated simultaneously within a 20 $\times 20 \mathrm{~cm}$ field. Whole-body irradiation was performed using $250 \mathrm{MeV}$ protons from the synchrotron accelerator housed at the Loma Linda University Medical Center, as described previously [24, 25]. Mice were irradiated at the entrance region of the Bragg curve, receiving a total dose of 1 Gy over approximately 100 seconds. Minimizing time between radiation and unloading was a consideration in selecting this dose rate, as was access to the synchrotron facility. 100 seconds was a compromise allowing for efficient delivery of radiation to many mice over a reasonable period of time. Mice were irradiated behind a 400 $\times 400 \mathrm{~mm}^{2}$ polystyrene phantom in order to harden the proton beam (i.e., remove lowenergy particles) and allow a uniform dose to be administered throughout the entire mouse. Dose calibration was performed using a Markus parallel plate ionization chamber (National Institute of Standards and Technology traceable). The calibration method in the International Commission on Radiation Units \& Measurements Report 59 [26] was used to convert the ionization signal to dose in water. The sham treatment involved identical handling, procedures, and timing as the irradiation group, although the beam was not turned on. After the mice were subjected to the irradiation or sham procedure, they were observed until they resumed normal posture and behavior. All mice were then shipped overnight to Clemson University and immediately subjected to hindlimb unloading as described below (approximately 30-33 hours after irradiation). One day after arrival, the baseline control group (BSL) was killed and tissues were collected (see the "Study Endpoint" section for details). 


\subsection{Hindlimb Suspension}

One day after the irradiation or sham procedure, one half of the IRR mice and one half of the NR mice were subject to hindlimb suspension (HLS). The remaining mice were kept as normally loaded controls (LC). This allocation produced the following five groups: baseline (BSL), non-irradiated and normally loaded (NR+LC), irradiated and normally loaded (IRR $+\mathrm{LC}$ ), non-irradiated and hindlimb suspension (NR+HLS), irradiated and hindlimb suspension (IRR+HLS) (Table 1).

HLS was performed using a modification of the Morey-Holton method, as described previously [2]. Mice were suspended at an approximately $30^{\circ}$ angle using a custom-built tail harness consisting of a $3 \times 25 \times 30 \mathrm{~mm}$ section of Akton ${ }^{\circledR}$ viscoelastic polymer padding (Action Products; Hagerstown, MD) surrounded by a $30 \mathrm{~mm}$ section of Tygon R-3603 plastic tubing with an $8 \mathrm{~mm}$ internal diameter (Saint-Gobain Performance Plastics; Northboro, Massachusetts). The tail harness was attached via steel wire to a swivel clip mounted on a guide wire running the length of the cage. Using this setup, mice were able to access all areas of the cage.

\subsection{Study Endpoint}

At the end of the four-week experimental period, all mice were anesthetized with inhalation isoflurane $(2 \%)$ and killed by exsanguination via cardiac puncture followed by cervical dislocation. Hindlimbs were removed and both tibias and femurs were cleaned of all nonosseous tissue. The left femur of each animal, required for mechanical testing and compositional analysis, was allowed to air-dry for 48 hours [27]. The right femur and tibia were fixed in a $10 \%$ neutral buffered formalin solution for 48 hours, rinsed with distilled water, and stored in $70 \%$ ethanol.

\subsection{Serum Analyses}

At sacrifice, samples of whole blood were collected by cardiac puncture. Blood was allowed to clot for 30 minutes. Serum was then separated via centrifugation at $1500 \mathrm{~g}$ for 10 minutes. Markers of bone turnover present in the serum were measured using enzyme-linked immunosorbent assay (ELISA) kits for the bone formation marker osteocalcin (BT-470, Biomedical Technologies, Inc.; Stoughton, MA) and the bone resorption marker tartrateresistant acid phosphatase 5b (TRAP5b) (SB-TR103, Immunodiagnostic Systems Inc.; Fountain Hills, AZ). All ELISA procedures were performed according to the manufacturers' protocols.

\subsection{Bone Microarchitectural Analyses}

Cortical and trabecular bone architecture was analyzed using microcomputed tomography ( $\mu$ CT80, Scanco Medical; Brüttisellen, Switzerland) with an isotropic voxel size of $10 \mu \mathrm{m}$. Trabecular microarchitecture was scanned immediately distal to the growth plate in the proximal tibia as well as immediately proximal to the growth plate in the distal femur. Analysis of trabecular bone was performed on 100 slices $(1.0 \mathrm{~mm}$ total), producing images for analysis. Bone morphometric parameters were then quantified using Scanco software. Trabecular bone parameters included: trabecular bone volume fraction (BV/TV), connectivity density (Conn.D), trabecular number (Tb.N), trabecular separation (Tb.Sp), and structural model index (SMI). Cortical analysis was performed immediately distal to the region of trabecular analysis in the tibia and immediately proximal to the region of trabecular analysis in the femur with 30 slices $(0.3 \mathrm{~mm})$ selected at each site. Cortical bone volume (Ct.BV), cortical total volume (Ct.TV), cortical porosity (Ct.Po), and polar moment of inertia (pMOI) were calculated from these sections. 


\subsection{Mechanical Testing}

In order simulate in vivo properties, the air-dried left femurs were rehydrated in phosphatebuffered saline for 90 minutes prior to evaluation [27]. Three-point bending tests were performed using an Instron 5582 (BlueHill 2 software, Instron Corp.; Norwood, MA). Femurs were tested to failure with a $9 \mathrm{~mm}$ span length and a deflection rate of $5 \mathrm{~mm} / \mathrm{min}$. All bones were tested in the same orientation: the single-point load was applied middiaphysis on the posterior surface. The maximal force $(\mathrm{N})$ and deflection $(\mathrm{mm})$ were measured for all mechanically tested bones. These two properties were also determined at the elastic limit $\left(\mathrm{P}_{\mathrm{e}}, \delta_{\mathrm{e}}\right)$ and the failure point. The $0.2 \%$ offset method was used to quantify yield properties. The ability of the femur to resist displacement (i.e., stiffness; $\mathrm{N} / \mathrm{mm}$ ) was calculated from elastic force/elastic deflection $\left(\mathrm{P}_{\mathrm{e}} / \delta_{\mathrm{e}}\right)$.

\subsection{Bone Mineral Composition}

Mineral-content analysis was performed on femurs fractured during mechanical testing. Prior to analysis, the enlarged ends of the femurs (epiphysis + metaphysis) were separated where distal and proximal metaphyses join the diaphysis. Mineral content data was obtained from these combined bone ends and also separately for the diaphysis. A properly calibrated analytical scale (Mettler Toledo UMT2; Columbus, $\mathrm{OH}$ ) was used for all measurements. Dry mass (DryM) was measured after heating the bones to $105^{\circ} \mathrm{C}$ for 24 hours. Mineral mass (MinM) was measured after the bones had been heated at $800^{\circ} \mathrm{C}$ for an additional 24 hours. Percent mineralization was calculated as: $\% \operatorname{Min}=(\operatorname{MinM}) /(\operatorname{DryM}) * 100$.

\subsection{Histology}

In order to examine the post-irradiation activity of bone-resorbing osteoclasts, right tibias from a subset of mice ( $n=6 /$ group) were selected for histological analyses. After fixation, tibias were decalcified in a weak formic acid solution (Immunocal ${ }^{\mathrm{TM}}$, Decal Chemical Corporation; Tallman, NY) for 2 days. Radiographs assessed the earliest time point of complete decalcification. Following this, tibias were embedded in a glycol methacrylate resin (Immunobed ${ }^{\mathrm{TM}}$, Polysciences; Warrington, PA) and cut into sagittal sections with a thickness of $1.5 \mu \mathrm{m}$. Osteoclast presence was determined by TRAP staining of the slides using a commercially available kit (Sigma; St. Louis, MO) and then counterstaining with hematoxylin (Sigma). Only TRAP-positive cells with two or more nuclei were counted as osteoclasts. Two sections from each bone were analyzed.

Analyses were performed using SigmaScan Pro Software (Systat Software, Inc., Richmond, CA). The evaluator was blinded to specimen origin and group assignment until after evaluation and analysis was complete. Histomorphometric evaluation was performed from captured micrographs $(400 \times)$ throughout the metaphysis, starting approximately $0.25 \mathrm{~mm}$ distal from the growth plate (in order to exclude the primary spongiosa) and extending a further $0.5 \mathrm{~mm}$. Bone histomorphometric parameters for the proximal metaphysis of the tibia were measured and reported in units specified by the American Society for Bone and Mineral Research Histomophometry Nomenclature Committee [28]. All surface measurements were quantified relative to total bone surface (BS). Histological measurements included osteoclast surface (Oc.S/BS; \%); eroded surface with the inclusion of osteoclast surface (surface covered by Howship's lacunae plus osteoclasts, [ES(Oc+)/BS], $\%$ ); and eroded surface with the exclusion of osteoclast surface (surface covered by Howship's lacunae, [ES(Oc-)/BS], \%). The number of osteoclasts (N.Oc) within the region of interest along trabeculae of the secondary spongiosa was also determined (N.Oc/BS, $\left.\mathrm{mm}^{-1}\right)$. 


\subsection{Statistics}

Statistical analysis of the experimental results was completed using SigmaStat software v3.5 (Systat Software Inc.; San Jose, CA). Comparisons were made using a two-way analysis of variance (ANOVA). Unless otherwise noted, no statistical interaction was present. Where significance was present overall for a treatment condition, a Student Newman-Keuls test was performed to reveal significance between specific groups (e.g., radiation within HLS, radiation within LC, or loading condition within NR). Statistical significance was set at P < 0.05 for all comparisons. Data are shown as mean \pm standard error (SE), unless otherwise indicated.

\section{RESULTS}

\subsection{Animal Mass}

Mean animal mass at sacrifice for each group was as follows: $\mathrm{BSL}=22.4 \pm 0.27 \mathrm{~g}, \mathrm{NR}+\mathrm{LC}=$ $23.2 \pm 0.32 \mathrm{~g}, \mathrm{IRR}+\mathrm{LC}=23.3 \pm 0.38 \mathrm{~g}, \mathrm{NR}+\mathrm{HLS}=22.6 \pm 0.30 \mathrm{~g}$, and IRR+HLS $=22.4 \pm 0.30$ $\mathrm{g}$. There was no significant effect of irradiation on animal mass. Overall, HLS mice were 3$4 \%$ lighter than LC mice. However, these differences were not significant when comparing individual groups (i.e., $\mathrm{NR}+\mathrm{HLS}$ versus $\mathrm{NR}+\mathrm{LC}$ and IRR+HLS versus IRR+LC).

\subsection{Baseline Microarchitectural Parameters}

Compared to 20 week-old NR+LC, 16-week-old baseline mice (BSL) had higher trabecular $\mathrm{BV} / \mathrm{TV}$ as well as higher overall trabecular bone quality in both the proximal tibia (Figure 1) and the distal femur (Figure 2), as represented by higher trabecular bone parameters including Conn.D, Tb.N, Tb.Sp, and SMI.

\subsection{Effects of Irradiation on Normally Loaded Mice}

Compared to NR+LC, IRR+LC mice experienced significant deterioration of trabecular microarchitectural parameters. In the proximal tibia, IRR+LC mice had significantly lower BV/TV (-16\%), Conn.D (-28\%), and Tb.N (-7.7\%) and significantly higher Tb.Sp (+9.0\%) and SMI $(+11 \%)$ (Figure 1). In the distal femur, IRR+LC had significantly lower BV/TV $(-22 \%)$ and Conn.D (-37\%) and higher SMI (+8.0\%) (Figure 2). Tb.N and Tb.Sp were unchanged.

In both the tibia and femur, cortical bone parameters, including Ct.TV, Ct.BV, Ct.Po, and pMOI were not different between IRR+LC and NR+LC (Table 2). Similarly, femurs from IRR+LC mice did not have significantly different mechanical strength or any mechanical parameters assessed when compared to NR+LC (Figure 3).

Percent mineralization in the femur was not significantly different between IRR+LC and NR $+\mathrm{LC}$ (Figure 4). Serum markers of bone turnover, including osteocalcin and TRAP5b, were also not different between IRR+LC and NR+LC (Table 3). In addition, histological parameters, including ES(Oc+)/BS, ES(Oc-)/BS, Oc.S/BS, and N.Oc/BS, were not significantly different between IRR+LC and NR+LC mice (Table 3).

\subsection{Effect of Unloading on Non-Irradiated Mice}

Compared to NR+LC, NR+HLS mice experienced substantial deterioration of trabecular bone. At the proximal tibia, NR+HLS mice were found to have significantly lower trabecular BV/TV (-74\%), Conn.D (-86\%), Tb.N (-22\%), and higher Tb.Sp (+29\%) and SMI $(+45 \%)$ (Figure 1). There were similar findings in the trabecular bone of the distal femur, where NR+HLS mice had significantly lower BV/TV (-60\%), Conn.D (-75\%), and Tb.N (-12\%) and higher Tb.Sp (+14\%) and SMI (+7.3\%) compared to NR+ LC (Figure 2). 
As opposed to irradiated mice, NR+HLS mice experienced significant loss of cortical microstructure when compared to NR+LC (Table 2). In the proximal tibia, these mice had significantly lower Ct.TV (-19\%), Ct.BV (-23\%), and pMOI (-18\%), and higher Ct.Po $(+34 \%)$. In the distal femur, NR+HLS mice had significantly lower Ct.TV $(-21 \%)$, Ct.BV $(-24 \%)$, pMOI $(-21 \%)$, and higher Ct.Po $(+33 \%)$ versus NR+LC. NR+HLS mice also had reduced mechanical competency compared to $\mathrm{NR}+\mathrm{LC}$, with significantly reductions in stiffness, maximum force, and elastic force (Figure 3). Percent mineralization was lower in both the epiphysis and diaphysis in NR+HLS versus NR+LC mice (Figure 4).

NR+HLS mice did not have significantly different levels of the bone formation marker osteocalcin when compared to levels in NR+LC (Table 3). However, NR+HLS mice did have significantly higher levels of the bone resorption marker TRAP5b when compared to NR+LC. Histological examination of the proximal tibia did not reveal any significant differences between NR+HLS and NR+LC mice in terms of Oc.S/BS or N.Oc/BS. Overall, HLS mice had higher ES(Oc+)/BS and ES(Oc-)/BS. However, these differences were not significant when individual groups were compared (i.e., $\mathrm{NR}+\mathrm{HLS}$ versus $\mathrm{NR}+\mathrm{LC}$ mice).

\subsection{Effect of Irradiation on Unloaded Mice}

Compared to NR+HLS, IRR+HLS mice had deteriorated trabecular microarchitecture. In the tibia, IRR+HLS mice had significantly lower Conn.D (-38.9\%) and Tb.N $(-8.0 \%)$ and significantly higher $\mathrm{Tb} . \mathrm{Sp}(+9.6 \%)$ and SMI $(+9.6 \%)$ when compared to NR+HLS (Figure 1). There was a trend of decreased BV/TV $(-17.4 \% ; \mathrm{P}=0.053)$ in IRR+HLS mice compared to NR+HLS (Figure 1). A statistically significant interaction between radiation and unloading was present for Conn.D in the tibia. In the femur, IRR+HLS mice had significantly lower BV/TV $(-26.4 \%)$ and Conn.D (-45.9\%) and significantly greater SMI $(+7.4 \%$ ) versus NR+HLS (Figure 2 ). In the femur, Tb.Sp and Tb.N were not significantly different between IRR+HLS and NR+HLS mice. The percent differences between irradiated and non-irradiated animals were similar for both the LC and HLS mice for several trabecular microarchitectural parameters in both the tibia and the femur (Table 4).

In both the tibia and femur, cortical bone parameters (including Ct.TV, Ct.BV, Ct.Po, and pMOI) were not different between IRR+HLS and NR+HLS mice (Table 2). Similarly, IRR + HLS did not have significantly different measures of mechanical strength when compared to NR+HLS (Figure 3).

While mineral content in the diaphysis of the femur was not significantly different, IRR +HLS mice had lower mineral content in the epiphysis and overall compared to NR+HLS (Figure 4). A statistically significant interaction between radiation and unloading was present for mineral content in the epiphysis and total femur. Markers for bone turnover, including osteocalcin and TRAP5b, were not significantly different between IRR+HLS and NR+HLS mice (Table 3). In addition, there was no significant difference in histological parameters, including ES(Oc+)/BS, ES(Oc-)/BS, Oc.S/BS, and N.Oc/BS, when comparing IRR+HLS and NR+HLS mice.

\section{DISCUSSION}

In the present study we have confirmed that $1 \mathrm{~Gy}$ of whole body proton irradiation results in significant loss of trabecular bone at the proximal tibia. We have also broadened the scope of bone loss associated with irradiation by demonstrating similar and significant deleterious effects on trabecular bone mass and microarchitecture at the distal femur. These results suggest that the effect of radiation on bone is not specific to one site. Previous studies from our laboratory have demonstrated bone loss in mice four months after proton radiation exposure [19]. Here, we report a similar amount of bone loss in the tibia $(\sim 15 \%)$ just one 
month after irradiation. This similarity suggests that the deterioration of bone resultant from $1 \mathrm{~Gy}$ of radiation is persistent long after exposure.

These findings may have significant implications for the recovery of astronauts following spaceflight radiation exposure as a prolonged period of compromised bone structure may increase the risk of fracture during a mission or following return to Earth. Models predicting recovery of skeletal sites such as the lumbar spine, pelvis, and calcaneus following 4-6 month missions on the ISS suggest that it would take nine months to regain $50 \%$ of the BMD lost during spaceflight [29]. Indeed, direct measurements on astronauts following long duration missions have shown that proximal femur bone mineral density (BMD) and calculated bone strength were only partially recovered a year after return to Earth [30]. It is clear that the recovery period is much longer than the actual period of spaceflight exposure and, as we have shown, exposure to types and doses of radiation found outside of low Earth orbit may further comprise bone structure and delay recovery.

It is important to note that the bone loss observed in the present study occurred in skeletally mature mice. While high-linear energy transfer (LET) spaceflight radiation has been demonstrated to lead to bone loss in growing mice [31], the effect of low-LET protons on bone in skeletally mature animals has not been previously studied. Since astronauts are themselves skeletally mature (i.e., aged $30-50$ years), this is a critical finding. Furthermore, these results suggest that the bone loss we observed is not likely due to changes in growth: three previous studies from our laboratory investigated the effect of low-LET proton irradiation in relatively young ( 8 to 9 -week-old) mice $[6,19,23]$. Bone loss in these studies was similar to what we observed presently. Thus, while the underlying mechanisms mediating radiation-induced bone loss may be different between growing and mature animals, the fact that similar bone loss occurred in these studies suggests otherwise. Bone volume fraction and other measures of trabecular bone quality were lower in control animals sacrificed at the end of the one-month study, compared to baseline mice. This "natural" bone loss is consistent with previous investigations characterizing the decline of trabecular-bone parameters with age [32-34]. These findings also indicate that the lower trabecular bone quality in irradiated and hindlimb suspended mice was not due to an inhibition of bone growth. Histological examination and measurements of serum-bone-turnover markers did not reveal any significant differences following irradiation in either the loaded control or hindlimb suspension groups. Indeed, the radiation effects that we observed at four weeks primarily consisted of lower structural and functional parameters, rather than an effect on osteoclast or osteoblast activity. These data, combined with other recent studies demonstrating that the skeletal effects of radiation are rapid and initiated by the activation of osteoclasts $[20,35]$, indicate that bone turnover likely stabilized by the four-week post irradiation time point when these assays were conducted. Future studies should implement blood collection and histological assays at multiple early time points to more completely characterize the effects on bone turnover and cellular mediators.

A four-week period of hindlimb suspension had significant deleterious effects on trabecular bone. Unloaded, nonirradiated mice had $74 \%$ and $60 \%$ lower trabecular bone volume fraction than normally loaded, nonirradiated mice in the tibia and femur, respectively, which is consistent with our previous experience with this model [36]. Given the approximate 1\% loss of bone mass per month documented in astronauts on the ISS [1], it is not likely that astronauts will encounter this degree of bone loss on a long-duration mission; furthermore, the degree of bone loss made it more difficult to measure further changes in certain bone parameters with the addition of irradiation. That is, the extended period of unloading may have effectively pushed the bone loss to its physiological maximum, thus preventing the observation of additional loss from irradiation. It is clear that HLS represents an accelerated model of bone loss compared to spaceflight; however, it has been well validated and remains 
the standard for ground-based modeling of microgravity unloading [2, 37, 38]. Despite the reduced resolution, combined effects of irradiation prior to unloading were still observed. In the tibia, reductions in connectivity density, structural-model index, trabecular separation, and trabecular number exhibited an additive effect when irradiation was combined with unloading. Furthermore, there was a statistical trend indicating additional loss of bone volume fraction in irradiated mice subjected to unloading $(\mathrm{P}=0.053)$. The effects of irradiation combined with unloading were additive for bone volume fraction, connectivity density, and structural-model index in the femur. Percent mineralization was also lower for the combination of irradiation and unloading, compared to unloading alone. Taken together, these findings support the conclusion that irradiation and unloading leads to greater skeletal deterioration than unloading alone. While there are important caveats in the translation of these findings to spaceflight applications, astronauts exposed to relatively high doses of radiation on missions beyond low-Earth orbit may be at risk for higher levels of bone loss than astronauts on the International Space Station, where the Earth's magnetic field offers greater natural protection from radiation exposure [5].

Relevant to the interpretation of these results is the presence of interactions between the two treatments. The only statistical interactions detected between irradiation and unloading were for the parameter of trabecular connectivity density in the tibia and mineral content. That is, for every other measured parameter, the effect of irradiation did not depend on whether the mouse was unloaded or not. Thus, even though the effect of four weeks of unloading was large, the effect of irradiation was statistically the same in both unloaded and normally loaded controls. This finding is supported by comparison of the relative effects of irradiation in normally loaded and unloaded mice (Table 2). In both the tibia and the femur, irradiation had similar effects across several trabecular parameters, providing additional evidence that the effect of irradiation and unloading on bone may be additive.

Our data demonstrate that four weeks of unloading results in substantial degradation and loss of trabecular bone. To the authors' knowledge, the greatest measured bone loss after hindlimb suspension of skeletally mature mice was 59\% in BALB/c mice following two weeks of unloading [3]. Depending on site and strain of mouse, other studies have noted bone loss on the order of 10-25\% following two-to-three weeks of unloading [4, 39, 40]. The much greater bone loss observed in the present study suggests that bone loss is occurring in the fourth week of unloading and thus does not appear to plateau during this period. While a shorter period of HLS may be able to better model the bone loss that is likely to occur in an astronaut on a four-to-six month mission, the high degree of loss in this longer period does raise notable questions regarding the possible degree of bone loss in astronauts on a two-to-three year mission. Modeling such a scenario would be further complicated by the period of reloading that would occur while the astronauts lived and worked on the surface of Mars, for example. Further study is needed to understand the skeletal effects of various degrees of unloading, reloading, and radiation exposure. Another important consideration in the study design is how it corresponds to an actual spaceflight situation. While the use of irradiation followed by unloading is the most logistically feasible study design, it is different than the scenario of an astronaut being exposed to a large amount of proton radiation from a solar particle event. In this case, astronauts would experience a potentially lengthy period of skeletal unloading prior to radiation exposure. Currently, it is not known how the timing of irradiation and unloading affects the skeletal response or degree of bone loss. This is also an area that requires further investigation.

The present study provides additional evidence to suggest that the skeletal effects of proton radiation are specific to the trabecular bone compartment. As expected, we observed modest loss of cortical bone following unloading; however, there was no effect of proton irradiation alone on cortical bone. In addition, there was no additional effect of combined irradiation 
and unloading on cortical microstructure (Table 3). These findings are corroborated by the lack of change in assessed mechanical strength parameters, which are highly dependent on cortical bone in the three-point bending test paradigm (Figure 3). Previous work by our group has not demonstrated an effect of proton radiation on cortical bone [6, 19, 23].

It is difficult to design an experiment that adequately mimics the types and doses of radiation that astronauts can expect to encounter in the spaceflight environment. For example, while a solar particle event has a high particle fluence compared to galactic cosmic rays, the total dose from such an event would be delivered over a period of hours-to-days instead of minutes, as in the current study. The effect of dose rate on the response of bone is unknown. It is known that the effects of radiation in nonosseous tissues are dramatically dependent on dose rate. The ability of the cell to initiate repair mechanisms is intimately related to its ability to recover from radiation-induced damage [12]. In general, a dose of radiation administered over a 1-minute period would produce far more damage than the same dose and kind over a 1-hour period. Indeed, this concept of cellular repair time (which includes DNA repair, free-radical scavenging, and replacement of damaged macromolecules) is the basis of the dose fractionation that guides the use of therapeutic radiation for cancer [12]. Because astronauts would be subject to both continuous exposure from low-dose radiation (such as that from galactic cosmic rays) and potentially short bursts of relatively high dose proton radiation (such as that from a solar particle event), it can be seen why design of adequate Earth-based models represent a logistical challenge. In addition, previous studies from our laboratory indicate that mixed radiation may have more of an effect on bone than a single type [31]. Thus, the effects of proton radiation on bone may be greater in the presence of other radiation types.

The results of the present study demonstrate that a 1 Gy dose of proton radiation has deleterious effects on trabecular bone in the tibia and femur of skeletally mature mice. Mechanical unloading induced significant loss of both trabecular and cortical bone. Investigation of the combined effect of proton irradiation followed by unloading suggests an additive effect for certain parameters. Indeed, for many indices, this combination produced significantly greater declines in bone structural properties. Statistical analysis revealed some of the effects of radiation were largely independent of unloading, suggesting that astronauts on long duration missions beyond the protective radiation shielding of low Earth orbit may be at greater risk for skeletal deterioration. Of course, further study is needed to refine models of the spaceflight environment (including variations in the timing of radiation and unloading, the period of unloading, and the dose, rate, and type of radiation) in order to better understand the risks to skeletal health and aid in the development of effective countermeasures.

\section{Acknowledgments}

The author's would like to acknowledge grant support from the National Space Biomedical Research Institute (BL01302 through NASA NCC 9-58) and NIH 5 R01 AR059221-02. Special thanks to Dr. Henry J Donahue (R01 AG013087) for partial funding support of SAL.

\section{REFERENCES}

1. Lang T, LeBlanc A, Evans H, Lu Y, Genant H, Yu A. Cortical and trabecular bone mineral loss from the spine and hip in long-duration spaceflight. J Bone Miner Res. 2004; 19:1006-1012. [PubMed: 15125798]

2. Morey-Holton ER, Globus RK. Hindlimb unloading rodent model: technical aspects. J Appl Physiol. 2002; 92:1367-1377. [PubMed: 11895999]

3. Judex S, Garman R, Squire M, Busa B, Donahue LR, Rubin C. Genetically linked site-specificity of disuse osteoporosis. J Bone Miner Res. 2004; 19:607-613. [PubMed: 15005848] 
4. Squire M, Donahue LR, Rubin C, Judex S. Genetic variations that regulate bone morphology in the male mouse skeleton do not define its susceptibility to mechanical unloading. Bone. 2004; 35:13531360. [PubMed: 15589216]

5. Reitz G. Characteristic of the radiation field in low Earth orbit and in deep space. Z Med Phys. 2008; 18:233-243. [PubMed: 19205293]

6. Hamilton SA, Pecaut MJ, Gridley DS, Travis ND, Bandstra ER, Willey JS, Nelson GA, Bateman TA. A murine model for bone loss from therapeutic and space-relevant sources of radiation. J Appl Physiol. 2006; 101:789-793. [PubMed: 16741258]

7. Benton ER, Benton EV. Space radiation dosimetry in low-Earth orbit and beyond. Nucl Instrum Methods Phys Res B. 2001; 184:255-294. [PubMed: 11863032]

8. Blakely EA. Biological effects of cosmic radiation: deterministic and stochastic. Health Phys. 2000; 79:495-506. [PubMed: 11045523]

9. Townsend LW. Implications of the space radiation environment for human exploration in deep space. Radiat Prot Dosimetry. 2005; 115:44-50. [PubMed: 16381680]

10. Benton ER, Benton E. Space radiation dosimetry in low-Earth orbit and beyond. Nuclear Instruments \& Methods in Physics Research Section B-Beam Interactions with Materials and Atoms. 2001; 184:255-294.

11. Stephens DL Jr, Townsend LW, Hoff JL. Interplanetary crew dose estimates for worst case solar particle events based on historical data for the Carrington flare of 1859. Acta Astronaut. 2005; 56:969-974. [PubMed: 15835055]

12. Hall, EJ.; Giaccia, AJ. Time, Dose, and Fractionation in Radiotherapy. In: Barrett, K., editor. Radiobiology for the Radiologist. 6th Edition. Philadelphia, USA: Lippincott Williams \& Wilkins; 2006. p. 378-406.

13. Setlow, R.; Dicello, JF. Radiation hazards to crews of interplanetary missions: biological issues and research strategies. Washington, D. C.: Space Studies Board, National Research Council, \& National Academy Press; 1996. p. 13-34.

14. Parsons JL, Townsend LW. Interplanetary crew dose rates for the August 1972 solar particle event. Radiat Res. 2000; 153:729-733. [PubMed: 10825747]

15. NASA. Strategic Plan. Wasington, D.C.: 2011. p. 10

16. Cucinotta FA, Wu H, Shavers MR, George K. Radiation dosimetry and biophysical models of space radiation effects. Gravit Space Biol Bull. 2003; 16:11-18. [PubMed: 12959127]

17. Ohnishi K, Ohnishi T. The biological effects of space radiation during long stays in space. Biol Sci Space. 2004; 18:201-205. [PubMed: 15858386]

18. Cucinotta, FA. Acute radiation risk and countermeasures for space radiation. In: Vazquez, ME., editor. NSBRI Medical Radiation Countermeasures Workshop: National Space Biomedical Research Institute. 2006.

19. Bandstra ER, Pecaut MJ, Anderson ER, Willey JS, De Carlo F, Stock SR, Gridley DS, Nelson GA, Levine HG, Bateman TA. Long-term dose response of trabecular bone in mice to proton radiation. Radiat Res. 2008; 169:607-614. [PubMed: 18494551]

20. Willey JS, Livingston EW, Robbins ME, Bourland JD, Tirado-Lee L, Smith-Sielicki H, Bateman TA. Risedronate prevents early radiation-induced osteoporosis in mice at multiple skeletal locations. Bone. 2010; 46:101-111. [PubMed: 19747571]

21. Yumoto K, Globus RK, Mojarrab R, Arakaki J, Wang A, Searby ND, Almeida EA, Limoli CL. Short-term effects of whole-body exposure to (56)fe ions in combination with musculoskeletal disuse on bone cells. Radiat Res. 2010; 173:494-504. [PubMed: 20334522]

22. Alwood JS, Yumoto K, Mojarrab R, Limoli CL, Almeida EA, Searby ND, Globus RK. Heavy ion irradiation and unloading effects on mouse lumbar vertebral microarchitecture, mechanical properties and tissue stresses. Bone. 2010; 47:248-255. [PubMed: 20466089]

23. Lloyd SA, Bandstra ER, Travis ND, Nelson GA, Bourland JD, Pecaut MJ, Gridley DS, Willey JS, Bateman TA. Spaceflight-relevant types of ionizing radiation and cortical bone: Potential LET effect? Adv Space Res. 2008; 42:1889-1897. [PubMed: 19122806]

24. Gridley DS, Pecaut MJ, Dutta-Roy R, Nelson GA. Dose and dose rate effects of whole-body proton irradiation on leukocyte populations and lymphoid organs: part I. Immunol Lett. 2002; 80:55-66. [PubMed: 11716966] 
25. Gridley DS, Pecaut MJ, Miller GM, Moyers MF, Nelson GA. Dose and dose rate effects of wholebody gamma-irradiation: II. Hematological variables and cytokines. In Vivo. 2001; 15:209-216. [PubMed: 11491015]

26. International Commission on Radiation Units and Measurements. Clinical proton dosimetry. Bethesda, Md.: International Commission on Radiation Units and Measurements; 1998.

27. Broz JJ, Simske SJ, Greenberg AR, Luttges MW. Effects of rehydration state on the flexural properties of whole mouse long bones. J Biomech Eng. 1993; 115:447-449. [PubMed: 8309241]

28. Parfitt AM, Drezner MK, Glorieux FH, Kanis JA, Malluche H, Meunier PJ, Ott SM, Recker RR. Bone histomorphometry: standardization of nomenclature, symbols, and units. Report of the ASBMR Histomorphometry Nomenclature Committee. J Bone Miner Res. 1987; 2:595-610. [PubMed: 3455637]

29. Sibonga JD, Evans HJ, Sung HG, Spector ER, Lang TF, Oganov VS, Bakulin AV, Shackelford LC, LeBlanc AD. Recovery of spaceflight-induced bone loss: bone mineral density after longduration missions as fitted with an exponential function. Bone. 2007; 41:973-978. [PubMed: 17931994]

30. Lang TF, Leblanc AD, Evans HJ, Lu Y. Adaptation of the proximal femur to skeletal reloading after long-duration spaceflight. J Bone Miner Res. 2006; 21:1224-1230. [PubMed: 16869720]

31. Bandstra ER, Thompson RW, Nelson GA, Willey JS, Judex S, Cairns MA, Benton ER, Vazquez ME, Carson JA, Bateman TA. Musculoskeletal changes in mice from 20-50 cGy of simulated galactic cosmic rays. Radiat Res. 2009; 172:21-29. [PubMed: 19580504]

32. Halloran BP, Ferguson VL, Simske SJ, Burghardt A, Venton LL, Majumdar S. Changes in bone structure and mass with advancing age in the male C57BL/6J mouse. J Bone Miner Res. 2002; 17:1044-1050. [PubMed: 12054159]

33. Glatt V, Canalis E, Stadmeyer L, Bouxsein ML. Age-related changes in trabecular architecture differ in female and male C57BL/6J mice. J Bone Miner Res. 2007; 22:1197-1207. [PubMed: 17488199]

34. Ferguson VL, Ayers RA, Bateman TA, Simske SJ. Bone development and age-related bone loss in male C57BL/6J mice. Bone. 2003; 33:387-398. [PubMed: 13678781]

35. Willey JS, Lloyd SA, Robbins ME, Bourland JD, Smith-Sielicki H, Bowman LC, Norrdin RW, Bateman TA. Early increase in osteoclast number in mice after whole-body irradiation with 2 Gy X rays. Radiat Res. 2008; 170:388-392. [PubMed: 18763868]

36. Lloyd SA, Travis ND, Lu T, Bateman TA. Development of a low-dose anti-resorptive drug regimen reveals synergistic suppression of bone formation when coupled with disuse. J Appl Physiol. 2008; 104:729-738. [PubMed: 18174391]

37. Morey-Holton ER, Globus RK. Hindlimb unloading of growing rats: a model for predicting skeletal changes during space flight. Bone. 1998; 22:83S-88S. [PubMed: 9600759]

38. Morey-Holton E, Globus RK, Kaplansky A, Durnova G. The hindlimb unloading rat model: literature overview, technique update and comparison with space flight data. Adv Space Biol Med. 2005; 10:7-40. [PubMed: 16101103]

39. Amblard D, Lafage-Proust MH, Laib A, Thomas T, Ruegsegger P, Alexandre C, Vico L. Tail suspension induces bone loss in skeletally mature mice in the C57BL/6J strain but not in the $\mathrm{C} 3 \mathrm{H} /$ HeJ strain. J Bone Miner Res. 2003; 18:561-569. [PubMed: 12619942]

40. Squire M, Brazin A, Keng Y, Judex S. Baseline bone morphometry and cellular activity modulate the degree of bone loss in the appendicular skeleton during disuse. Bone. 2008; 42:341-349. [PubMed: 17997144] 


\section{HIGHLIGHTS}

We examined the effects of mechanical unloading and radiation on bone in mice.

1 Gy of proton irradiation caused modest loss of trabecular, but not cortical bone.

4 weeks of unloading caused substantial loss of trabecular and cortical bone.

Combined radiation with unloading resulted in additive effects for many parameters. 
A

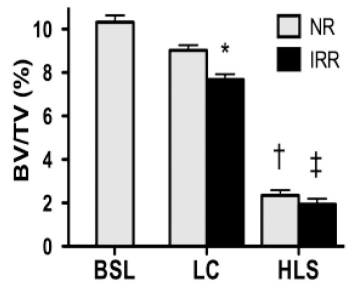

B

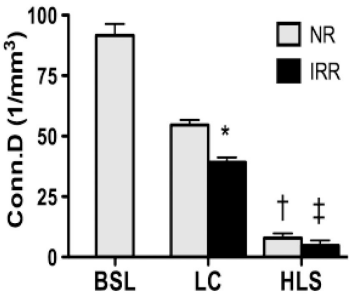

C

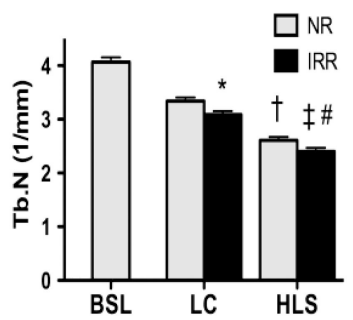

๑

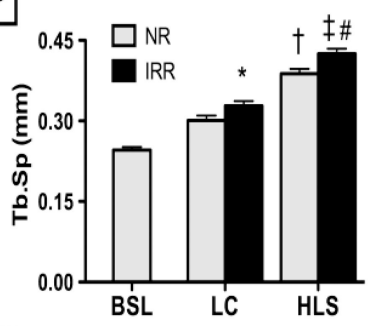

E

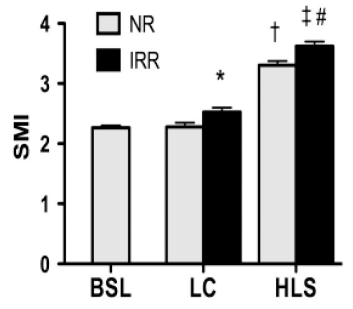

Figure 1.

Trabecular bone parameters obtained via MicroCT of the proximal tibia following $1 \mathrm{~Gy}$ proton irradiation and one month of unloading via hindlimb suspension. Trabecular Bone parameters assessed included: (A) Bone volume fraction (BV/TV); (B) Connectivity Density (Conn.D); (C) Trabecular Number (Tb.N); (D) Trabecular Separation (Tb.Sp); (E) Structure Model Index (SMI). BSL = Baseline; $\mathrm{LC}=$ normally loaded control; HLS $=$ hindlimb suspension; $\mathrm{NR}=$ non-irradiated; IRR $=$ irradiated. Data reported as mean $\pm \mathrm{SE}$.

Significance $(\mathrm{P}<0.05)$ denoted by $(*)$ for IRR $+\mathrm{LC}$ versus NR+LC, $(\dagger)$ for NR+HLS versus $\mathrm{NR}+\mathrm{LC}$, ( ) for IRR+HLS versus IRR+LC, and (\#) for IRR+HLS versus NR+HLS. 
A

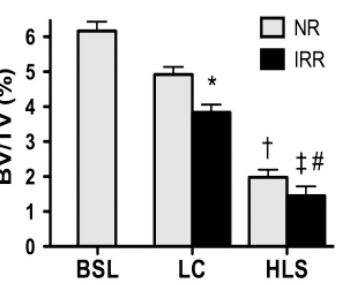

B

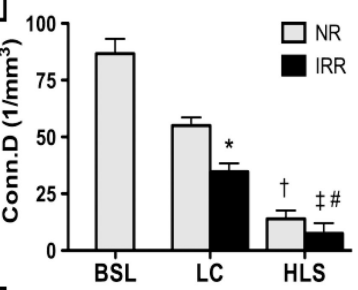

C

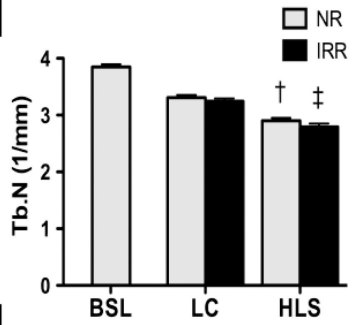

D

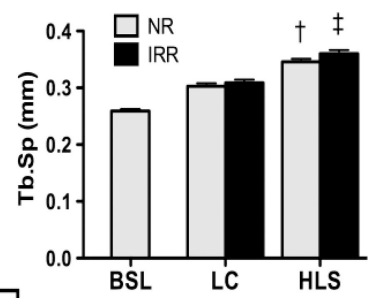

E

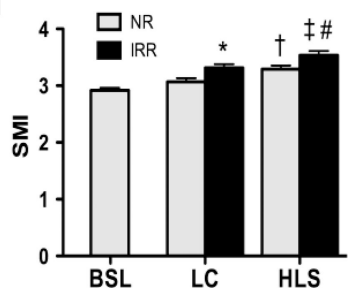

Figure 2.

Trabecular bone parameters obtained via MicroCT of the distal femur following 1 Gy proton irradiation and one month of unloading via hindlimb suspension. Trabecular Bone parameters assessed included: (A) Bone volume fraction (BV/TV); (B) Connectivity Density (Conn.D); (C) Trabecular Number (Tb.N); (D) Trabecular Separation (Tb.Sp); (E) Structure Model Index (SMI). BSL = Baseline; $\mathrm{LC}=$ normally loaded control; $\mathrm{HLS}=$ hindlimb suspension; $\mathrm{NR}=$ non-irradiated; IRR $=$ irradiated. Data reported as mean $\pm \mathrm{SE}$. Significance $(\mathrm{P}<0.05)$ denoted by $(*)$ for IRR+LC versus NR+LC, $(\dagger)$ for NR+HLS versus $\mathrm{NR}+\mathrm{LC}$, ( ) for IRR+HLS versus IRR+LC, and (\#) for IRR+HLS versus NR+HLS. 

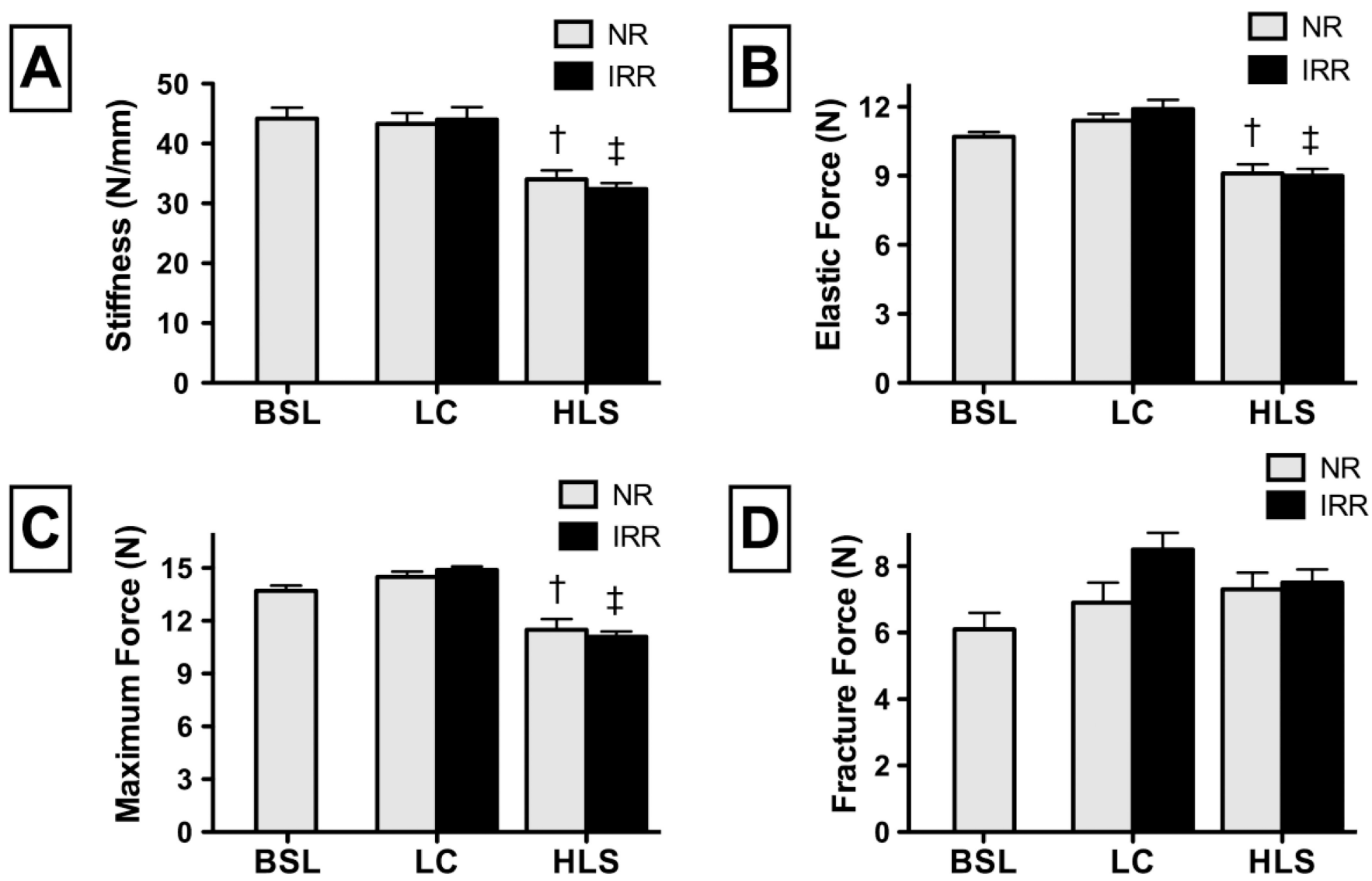

Figure 3.

Mechanical strength of the femur obtained via three-point bending following 1 Gy proton irradiation and one month of unloading via hindlimb suspension. Measured parameters included: (A) Stiffness; (B) Elastic Force; (C) Maximum Force; (D) Fracture Force. BSL = Baseline; $\mathrm{LC}=$ normally loaded control; HLS $=$ hindlimb suspension; $\mathrm{NR}=$ non-irradiated; $\mathrm{IRR}=$ irradiated. Data reported as mean $\pm \mathrm{SE}$. Significance $(\mathrm{P}<0.05)$ denoted by $(*)$ for IRR $+\mathrm{LC}$ versus NR+LC, $(\dagger)$ for NR+HLS versus NR+LC, $(\dagger)$ for IRR+HLS versus IRR $+\mathrm{LC}$, and (\#) for IRR+HLS versus NR+HLS. 

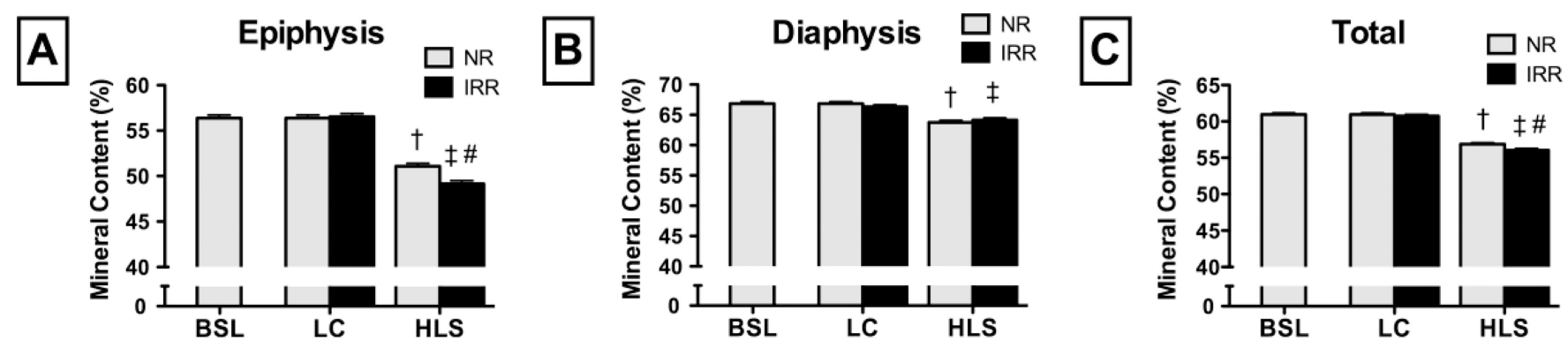

Figure 4.

Mineral content of the femur following $1 \mathrm{~Gy}$ proton irradiation and one month of unloading via hindlimb suspension. Mineralization was determined for the (A) Epiphysis; (B)

Diaphysis; $(\mathrm{C})$ Total Femur. BSL = Baseline; $\mathrm{LC}=$ normally loaded control; $\mathrm{HLS}=$ hindlimb suspension; $\mathrm{NR}=$ non-irradiated; $\mathrm{IRR}=$ irradiated. Data reported as mean $\pm \mathrm{SE}$. Significance $(\mathrm{P}<0.05)$ denoted by $(\dagger)$ for NR+HLS versus NR+LC, $(\ddagger)$ for IRR+HLS versus IRR+LC, and (\#) for IRR+HLS versus NR+HLS. 
Table 1

Study Design Combining Irradiation and Hindlimb Suspension

\begin{tabular}{ccc}
\hline & Non-Irradiatedx (NR) & Irradiated (IRR) \\
\hline Baseline (BSL) & BSL $(\mathrm{n}=16)$ & \\
Normally Loaded (LC) & NR+LC $(\mathrm{n}=17)$ & IRR+LC $(\mathrm{n}=17)$ \\
Hindlimb Suspended (HLS) & NR+HLS $(\mathrm{n}=17)$ & IRR+HLS $(\mathrm{n}=15)$ \\
\hline
\end{tabular}

Bone. Author manuscript; available in PMC 2013 October 01. 


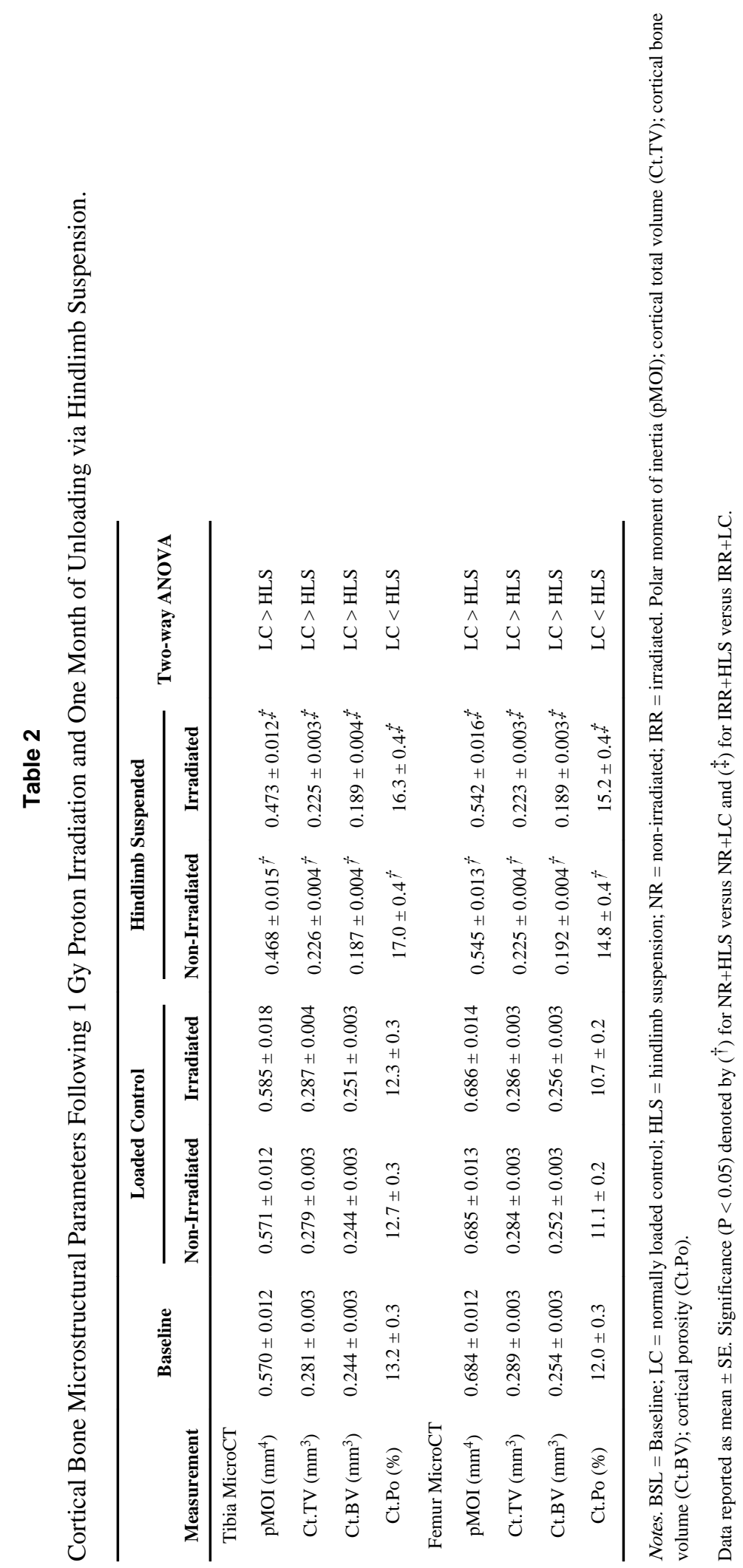




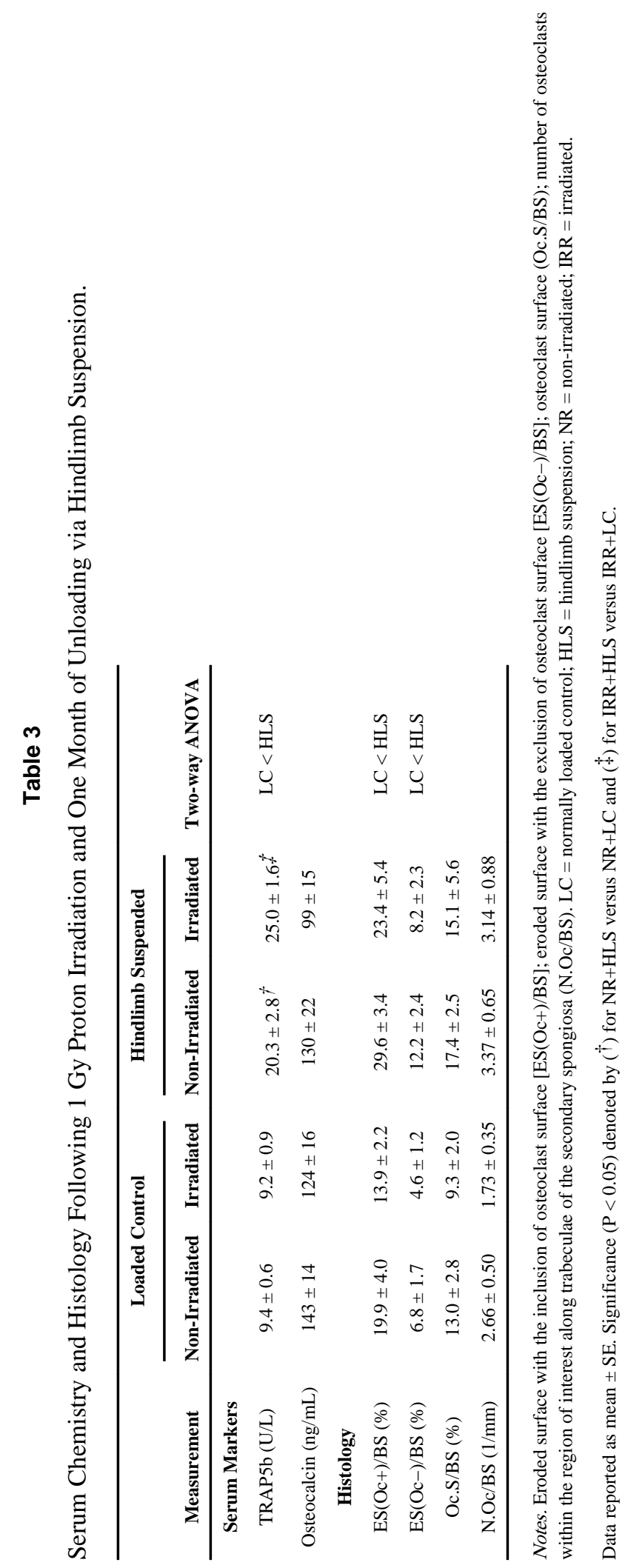

Bone. Author manuscript; available in PMC 2013 October 01. 
Table 4

Relative Differences in the Tibia and Femur Following 1 Gy Proton Irradiation and One Month of Unloading via Hindlimb Suspension.

\begin{tabular}{|c|c|c|c|c|}
\hline & \multicolumn{2}{|c|}{ Tibia } & \multicolumn{2}{|c|}{ Femur } \\
\hline & $\begin{array}{l}\text { Irradiation Effect in } \\
\text { Loaded Control (\%) }\end{array}$ & $\begin{array}{c}\text { Irradiation Effect in } \\
\text { Hindlimb Suspended (\%) }\end{array}$ & $\begin{array}{l}\text { Irradiation Effect in } \\
\text { Loaded Control }(\%)\end{array}$ & $\begin{array}{c}\text { Irradiation Effect in } \\
\text { Hindlimb Suspended (\%) }\end{array}$ \\
\hline $\mathrm{BV} / \mathrm{TV}$ & $-14.8^{*}$ & $-17.4^{+}$ & $-21.9^{*}$ & $-26.4^{*}$ \\
\hline Conn.D & $-28.3^{*}$ & $-38.9^{*}$ & $-37.0^{*}$ & $-45.9^{*}$ \\
\hline Tb.N & $-7.7^{*}$ & $-8.0 *$ & -1.9 & -3.9 \\
\hline Tb.Sp & $9.0^{*}$ & $9.6^{*}$ & 1.9 & 3.8 \\
\hline SMI & $10.9^{*}$ & $9.6^{*}$ & $8.0^{*}$ & $7.4^{*}$ \\
\hline
\end{tabular}

Notes. Bone volume fraction (BV/TV); connectivity density (Conn.D); trabecular number (Tb.N); trabecular separation (Tb.Sp); structure model index (SMI). BSL = Baseline; LC = normally loaded control; HLS = hindlimb suspension; NR = non-irradiated; IRR = irradiated.

Significant difference $(\mathrm{P}<0.05)$ from corresponding non-irradiated control denoted by $(*)$ as determined by two-way ANOVA followed by t-test comparing individual groups (IRR+LC vs NR+LC and IRR+HLS vs NR+HLS).

(+) denotes non-significant difference $(\mathrm{P}=0.053)$. 